\title{
ЭЛЕКТРОМИОСТИМУЛЯЦИЯ КАК АЛЬТЕРНАТИВА ФИЗИЧЕСКОЙ ТРЕНИРОВКЕ У ПАЦИЕНТОВ С ХОБЛ
}

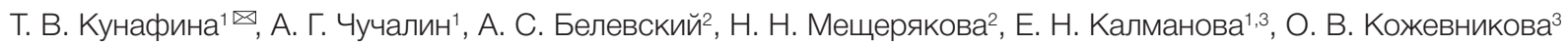

${ }^{1}$ Кафедра госпитальной терапии, педиатрический факультет,

Российский национальный исследовательский медицинский университет имени Н. И. Пирогова, Москва

2 Кафедра пульмонологии, факультет дополнительного профессионального образования,

Российский национальный исследовательский медицинский университет имени Н. И. Пирогова, Москва

${ }^{3}$ Городская клиническая больница имени Д. Д. Плетнева, Москва

\begin{abstract}
Пациенты с обострением хронической обструктивной болезни легких (ХОБЛ) не способны выполнять тренировочные упражнения в рамках программы легочной реабилитации. Альтернативой служит метод электромиостимуляции (ЭМС), поскольку его применение не вызывает усиления одышки у пациента. Целью работы была оценка эффективности краткосрочной ЭМС четырехглавой мышцы бедра на двигательную активность у пациентов с ХОБЛ. В проспективное открытое рандомизированное исследование вошли 36 пациентов, разделенные на две сопоставимые группы. Пациентам в основной группе проводили ЭМС в течение 10 дней. На 10-е сутки регистрировали и сравнивали клинико-функциональные параметры и потенциальные побочные эффекты. Между двумя группами не было отмечено существенных различий в отношении исходных характеристик. По результатам межгруппового анализа, основная группа имела статистически значимые улучшения показателей измерений, выполненных шагомерм и при миограсрии, равных соответственно 418,5 (86,0; 815,0) против 226,7 (48,0; 660,0) (p = 0,02), 463,0 (122; 804) против 210,5 (64; 481) ( $p=0,0001)$. Отмечалось значительное снижение баллов при оценке ХОБЛ по САТ-тесту и оценке одышки по $\mathrm{mMRC-}$ шкале и по шкале Borg: 22,8 (18,0; 34,0) против 28,4 (26,0; 34,0) ( $p=0,00007), 2,7$ (2,0; 4,0) против 3,1 (3,0; 4,0) $(p=0,03)$ и $6,3(5,0 ; 7,0)$ против $7,2(6,0 ; 9,0)(p=0,0002)$ соответственно. Побочных эффектов в основной группе отмечено не было. На основании полученных результатов можно сделать вывод, что краткосрочная ЭМС четырехглавой мышцы бедра улучшает двигательную активность пациентов, повышая качество жизни и способность выполнять программы легочной реабилитации в последующем, и является альтернативой физическим тренировкам у пациентов с ХОБЛ.
\end{abstract}

Ключевые слова: обострение ХОБЛ, дисфункция скелетной мускулатуры, легочная реабилитация, электромиостимуляция

$\checkmark$ Для корреспонденции: Татьяна Викторовна Кунасина

ул. Островитянова, д. 1, г. Москва, 117997; tana_07@mail.ru

Статья получена: 19.05.2018 Статья принята к печати: 25.07.2018

DOI: $10.24075 /$ vrgmu.2018.035

\section{NEUROMUSCULAR ELECTRICAL STIMULATION AS AN ALTERNATIVE TO PHYSICAL EXERCISE IN PATIENTS WITH COPD}

Kunafina $\mathrm{TV}^{1} \otimes$, Chuchalin $A G^{1}$, Belevsky $A S^{2}$, Mescheryakova $N^{2}{ }^{2}$, Kalmanova EN ${ }^{1,3}$, Kozhevnikova OV ${ }^{3}$

Department of In-Patient Care, Faculty of Pediatrics,

Pirogov Russian National Research Medical University, Moscow

2Department of Pulmonology, Faculty of Continuing Professional Education,

Pirogov Russian National Research Medical University, Moscow

${ }^{3}$ Pletnev City Clinical Hospital, Moscow

Patients with chronic obstructive pulmonary disease (COPD) are unable to do physical exercises included into standard pulmonary rehabilitation programs. Neuromuscular electrical stimulation (NMES) is a good alternative for such patients as it does not aggravate shortness of breath. The aim of this work was to assess the effect of short-term NMES of the quadriceps femoris muscle on the physical activity of patients with COPD. Our prospective open randomized study was carried out in 36 patients distributed into two groups. The main group was administered NMES for 10 days. On day 10 clinical and functional parameters, as well as adverse events, were evaluated. On admission to hospital, the groups did not differ in terms of the studied parameters. Following the treatment course, the main group significantly improved their step count and electromyography results $(418.5$ (86.0; 815.0) vs. 226.7 (48.0; 660.0), $p=0.02$, and 463.0 (122; 804) vs. 210.5 (64; 481 ), $p=0.0001$, respectively). The patients scored much less on the Mmrc and Borg scales and the CAT-test: 22.8 (18.0; 34.0) vs. 28.4 (26.0; 34.0), $p=0.00007 ; 2.7$ (2.0; 4.0) vs. 3.1 (3.0; 4.0), $p=0.03$; and 6.3 (5.0; 7.0) vs. 7.2 (6.0; 9.0), $p=0.0002$, respectively. No adverse events were registered in the main group. Based on the obtained results, we conclude that shortterm NMES of the quadriceps femoris muscle improves physical activity, the quality of life and ability to do physical exercise in patients with COPD providing them with a good alternative to standard rehabilitation programs.

Keywords: COPD exacerbation, skeletal muscle dysfunction, pulmonary rehabilitation, neuromuscular electrical stimulation

$\square$ Correspondence should be addressed: Tatiana V. Kunafina

Ostrovityanova 1, Moscow, 117997; tana_07@mail.ru

Received: 19.05.2018 Accepted: 25.07.2018

DOI: 10.24075/brsmu.2018.035 
Хроническая обструктивная болезнь легких (ХОБЛ) является одной из ведущих причин заболеваемости и смертности во всем мире [1, 2]. Естественное течение заболевания отмечено обострениями, которые влияют на прогноз у таких пациентов [2]. Становится все более очевидным, что ХОБЛ присущ ряд системных проявлений, особенно при тяжелом протекании заболевания, и что эти проявления оказывают выраженное влияние на выживаемость пациентов и развитие сопутствующих заболеваний. У пациентов с тяжелой формой ХОБЛ часто отмечается кахексия. Возможна потеря мышечной массы и слабость мышц в результате активации апоптоза и/или бездействия мышц $[3,4]$. При обострении заболевания отмечается увеличение дыхательной недостаточности, а также возрастает потребность в системных глюкокортикостероидах, при этом усугубляется и без того плохое состояние скелетной мускулатуры. Это, в свою очередь, приводит к утомлению дыхательной мускулатуры с одной стороны, и к ограничению двигательной активности - с другой. При уменьшении двигательной активности отмечается гиподинамия, приводящая $\mathrm{k}$ дистросии, а в последующем - к атросии скелетных мышц (рис. 1). Одной из самых крупных мышц, участвующих в двигательной активности является четырехглавая мышца бедра. Слабость и атрофия именно этой мышцы приводят к худшим прогнозам и повышению смертности у пациентов с ХОБЛ [4].

Из-за развивающейся слабости и атрофии скелетной и дыхательной мускулатуры у больных с тяжелой формой ХОБЛ, в терапии таких пациентов отводится большое значение физической тренировке $[5,6]$. Физические тренировки - основной компонент легочной реабилитации. Продолжительность тренировок может достигать 4-12 недель, оптимальным сроком считают 6-8 недель [6].
Легочная реабилитация на протяжении не менее 4 недель приводит к улучшению клинически и статистически значимых показателей качества жизни, в том числе уменьшению одышки, усталости, улучшению эмоционального статуса [7]. Однако пациенты с тяжелой и крайне тяжелой степенью ХОБЛ не способны к выполнению данных методов реабилитации из-за выраженной дыхательной недостаточности, общей слабости, низкой готовности к легочной реабилитации. В качестве альтернативы и «мостиком» к физическим тренировкам является электромиостимуляция [7]. Показана эффективность электромиостимуляции четырехглавой мышцы бедра, проводившейся на протяжении не менее 4 недель, в качестве оценки которой использовали шкалы по определению одышки и опросник Святого Георгия (St. George's Respiratory Questionnaire) [8].

Целью нашего исследования была оценка эффективности краткосрочного воздействия электромиостимуляции на четырехглавую мышцу бедра с применением шагомера и метода поверхностной электромиографии у пациентов с тяжелым течением и обострением ХОБЛ, не способных к выполнению стандартных программ легочной реабилитации.

\section{ПАЦИЕНТЫ И МЕТОДЫ}

В период с сентября 2016 г. по февраль 2018 г. В пульмонологическом отделении университетской клиники проводили открытое проспективное когортное рандомизированное сравнительное исследование. Обследовали 56 пациентов с обострением ХОБЛ, сопровождающимся духательной недостаточностью. Из них только у 36 пациентов была зафиксирована дисфункция четырехглавой мышцы бедра. Пациенты были разделены на две группы. В основной группе $(n=18)$ проводили
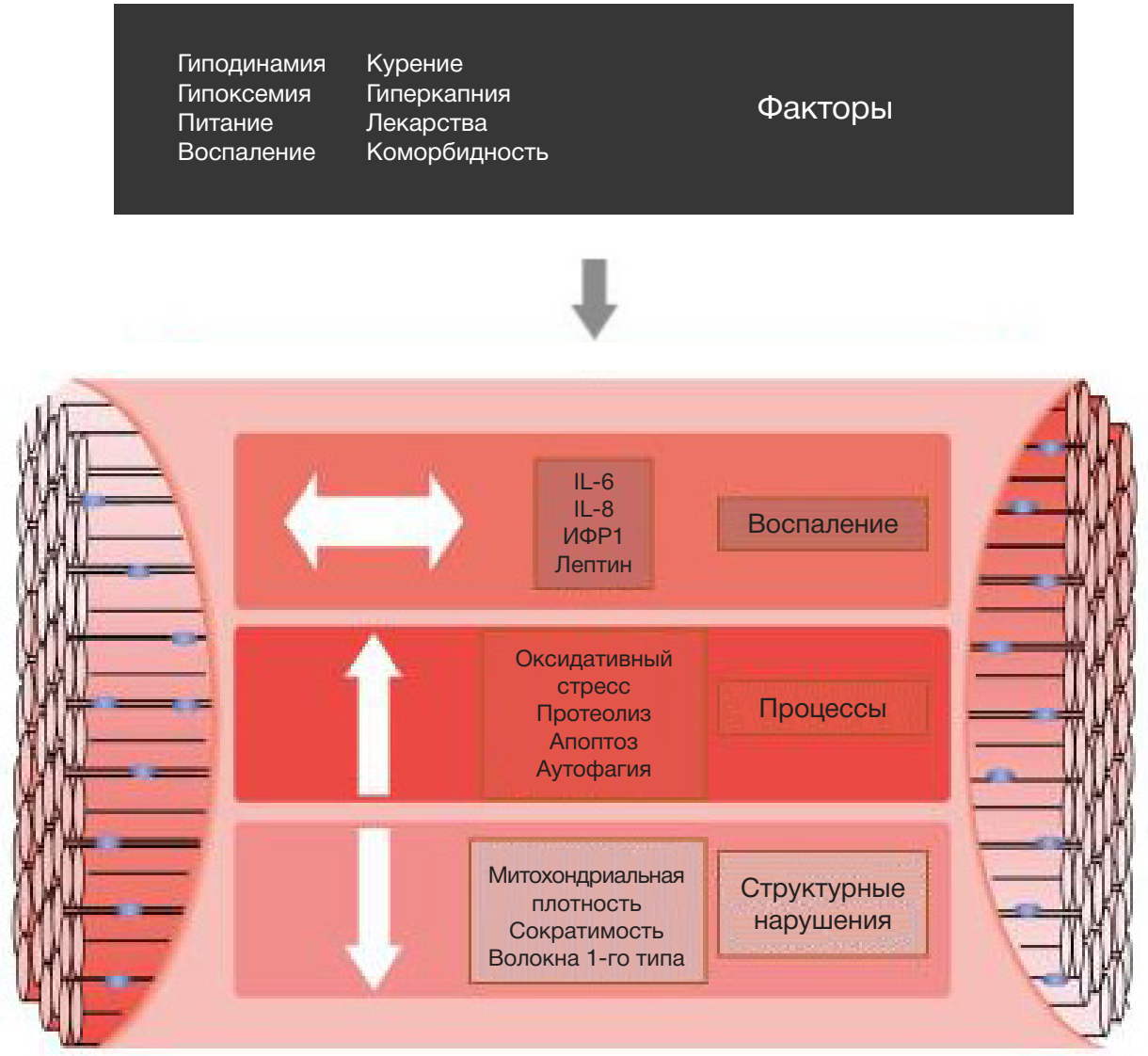

Рис. 1. Факторы, влияющие на повреждение мышечного волокна (По данным: Abdulai R. M. et al., 2017 [15]) 
краткосрочную электромиостимуляцию с помощью аппарата Compex (Compex, Франция). Результаты сопоставляли попарно с контрольной группой $(n=18)$. Критериями включения пациентов были обострение ХОБЛ, определяемое клинически по наличию не менее двух признаков и симптомов: нарастанию одышки, усилению кашля, увеличению количества или гнойности мокроты; признаки дыхательной недостаточности, сопровождающиеся слабостью, ограничением физической активности, подтвержденным результатами использования шагомера и метода электромиографии; дисфункция четырехглавой мышцы бедра (амплитуда при максимальном мышечном сокращении по данным поверхностной миографии <600 мкВ). Критерии исключения из исследования: гипертермия до фебрильных и субфебрильных значений температуры; отсутствие изменений на электромиографии; наличие пневмонии; психические нарушения, препятствующие установлению продуктивного контакта между врачом и пациентом; абсолютные противопоказания к применению аппарата, т. е. наличие кардиостимулятора, эпилепсия, серьезные нарушения артериального кровоснабжения нижних конечностей, наличие у пациента брюшной или паховой грыжи. Проведение исследования было одобрено локальным этическим комитетом (протокол №154 от 11.04.2016 г.). От всех пациентов было получено письменное информированное согласие на участие в исследовании.

Пациентов сопоставляли по возрасту, данным опросника mMRC (опросник Британского медицинского исследовательского совета для оценки тяжести одышки), САТ-тесту оценки ХОБЛ, спирометрическим параметрам (объему форсированного выдоха за 1 с $\left(О Ф \mathrm{~B}_{1}\right)$, соотношению ОФВ,/ФЖЕЛ), параметрам газового анализа артериальной крови (ГАК), а именно рН, парциальному давлению кислорода в артериальной крови $\left(\mathrm{PaO}_{2}\right)$, парциальному давлению углекислого газа в артериальной крови $\left(\mathrm{PaCO}_{2}\right)$, показателям электромиографии на момент поступления. Все пациенты получали стандартное лечение, назначаемое при обострении ХОБЛ в соответствии с международными рекомендациями (GOLD, 2017): ингаляционные бронхолитики, системные глюкокортикостероиды (преднизолон 20-40 мг в день) и эмпирическую антибактериальную терапию в соответствии с местной бактериальной устойчивостью и сопутствующими заболеваниями. Клинические параметры, показатели шагомера, электромиографии, шкалы одышки по Borg, mMRC, CAT, потенциальные побочные эффекты оценивали по истечении 10 дней и затем сравнивали между двумя группами.

\section{Электромиография}

Всем больным из основной группы и группы контроля проводили поверхностную электромиографию (ЭМГ) метод клинической электромиографии для исследования накожной суммарной биоэлектрической активности мышц в покое и при различных режимах напряжения согласно инструкции производителя. Электромиографию проводили с помощью многофункционального аппарата Nemus 1 (ЕB Neuro; Италия) с возможностью проведения ЭМГ и вызванных потенциалов.

\section{Забор крови для ГАК}

Забор крови производили с помощью автоматически наполняющихся шприцев (PICO70 ${ }^{\circledR}$ Radiometer; Дания) из лучевой артерии не ранее чем через 15 мин после прекращения оксигенотерапии и анализировали с помощью анализатора крови RAPIDLab 1200 Systems (Siemens; Германия) согласно инструкции производителя.

\section{Шагомер}

Физическую активность оценивали с помощью шагомера Torneo A-946BTRN (Compus pro; Китай). Аппарат для оценки количества шагов располагался на поясе одежды в течение 6 ч бодрствования.

\section{Электромиостимуляция}

Пациентам основной группы проводили электромиостимуляцию. Во время проведения процедуры пациент находился в положении сидя, либо в положении лежа на спине с валиком под коленями (пациенты с крайне тяжелой формой ХОБЛ). (+)-Электрод накладывали на кожу бедра на уровне моторной точки мышцы, где происходило наилучшее сокращение мышцы в условиях максимального комфорта. (-)-Электрод накладывали дистальнее на 10 см. Использовали чередование программ Sport| resistance (спорт, сопротивление), время работы аппарата 32 мин, и Aesthetic, firing (эстетика, укрепление), время работы аппарата 22 мин, через день. Интенсивность подбирали индивидуально по переносимости и ответному мышечному сокращению, в диапазоне от 10 до 35 мА.

\section{Статистический анализ}

Статистический анализ проводили с помощью программы Staistica 10 StatSoft. Использовали непараметрические методы описательной статистики: рассчитывали медианы $(\mathrm{Me})$, верхний $\left(Q_{3}\right)$ и нижний $\left(Q_{1}\right)$ квартили. Данные представляли в виде $\left(\mathrm{Me}\left(\mathrm{Q}_{1} ; \mathrm{Q}_{3}\right)\right)$. Сравнение двух независимых групп количественных признаков проводили с применением U-теста Манна-Уитни. Статистически достоверными считали различия при $p<0,05$.

\section{РЕЗУЛЬТАТЫ ИССЛЕДОВАНИЯ}

\section{Основные характеристики больных}

При поступлении пациенты обеих групп имели одинаковые основные характеристики, спирометрические данные и показатели миографии. Сравнение значений параметров ГАK $\left(\mathrm{pH}, \mathrm{PaO}_{2}, \mathrm{PaCO}_{2}\right)$ тоже не выявило существенной разницы (табл. 1).

\section{Влияние электромиостимуляции на качество жизни}

При анализе влияния ЭМС на качество жизни у пациентов основной группы выявлено улучшение показателей по CAT-тесту, шкале mMRC и шкале Borg. При этом в группе контроля значимого улучшения не выявлено (табл. 2).

\section{Влияние электромиостимуляции на клинические показатели}

Анализ влияния ЭМС на клинические параметры показал, что в обеих группах (основной и группе сравнения) заметно улучшились значения показателя $\mathrm{SpO}_{2}$, при этом на 10-й день в основной группе и группе сравнения достоверной разницы между значениями $\mathrm{SpO}_{2}$ не было установлено (табл. 2). 


\section{Влияние электромиостимуляции на двигательную активность и состояние четырехглавой мышцы бедра}

Внутригрупповой анализ показателей миографии и результатов измерений, сделанных шагомером, у пациентов основной группы показал их значительное улучшение. По результатам межгруппового анализа статистически значимое улучшение этих показателей наблюдалось у пациентов основной группы (рис. 2 и рис. 3 соответственно) на 10-й день (табл. 2).

\section{ОБСУЖДЕНИЕ РЕЗУЛЬТАТОВ}

Реабилитация пациентов с ХОБЛ имеет большое значение при комплексной терапии. Именно физическим тренировкам отводится при этом немаловажное значение. Эфффективность легочной реабилитации у пациентов с ХОБЛ имеет большой уровень доказательности [2]. Однако встает вопрос о том, как помочь тем пациентам, которые не способны к выполнению стандартных программ легочной реабилитации. По литературным данным, альтернативой физическим упражнениям может стать метод электромиостимуляции.

K настоящему времени проведено небольшое число клинических исследований, посвященных изучению эффективности электромиостимуляции четырехглавой мышцы бедра как одного из вариантов легочной реабилитации пациентов с тяжелой и крайне тяжелой формами ХОБЛ [7-10]. В представленных работах электромиостимуляция проводилась в достаточно длительные сроки, около 4-6 недель, и имела положительный эффект [11-14]. В качестве оценки использовали преимущественно субъективные методы, такие как, шестиминутный тест, опросник святого Георгия [8]. Результаты нашего иссследования показывают, что электростимуляция четырехглавой мышцы бедра в короткие сроки тоже приводит к улучшению функции указанной мышцы. Наиболее достоверным критерием оценки эффективности электростимуляции являются результаты электромиограсрии. В нашей работе при оценке влияния электростимуляции на насыщение гемоглобина капиллярной крови кислородом отмечено повышение $\mathrm{SpO}_{2}$, как в основной группе, так и в группе сравнения, что, вероятнее всего, обусловлено параллельно проводимой терапией у данных пациентов: приемом бронхолитических препаратов, кислородотерапией, у отдельных пациентов респираторной поддержкой. При оценке влияния электростимуляции на субъективное состояние пациента, а именно при оценке результатов измерения одышки по шкале Borg во время физической нагрузки и шкале mMRC, показано достоверное улучшение самочувствия у пациентов основной группы, вероятно, за счет уменьшения одышки на фоне улучшения функциональных свойств скелетной мускулатуры. Кроме того, прослеживается достоверное уменьшение количества баллов по вопроснику САТ, что связано с повышением двигательной активности пациента и улучшением качества жизни [7].

В нашем исследовании все субъективные изменения подтверждаются достоверным улучшением показателей миографии и результатов измерений, сделанных шагомером, использовавшихся нами в качестве оценки эффективности.

таблица 1. Основные характеристики групп

\begin{tabular}{|c|c|c|c|}
\hline Показатель & Основная группа ( $n=18)$ & Группа контроля $(n=18)$ & $p$ \\
\hline Возраст, лет & $66(53 ; 77)^{\star}$ & $69,6(53 ; 80)$ & $>0,05$ \\
\hline Пол, м/ж & $16 / 2$ & $15 / 3$ & $>0,05$ \\
\hline Индекс массы тела, кг/м² & $24,0(18,3 ; 31,2)$ & $22,9(18,1 ; 27,6)$ & $>0,05$ \\
\hline Индекс курящего человека (для курящих пациентов), пачка/лет & $45,9(30 ; 60)$ & $43,6(20 ; 60)$ & $>0,05$ \\
\hline САТ-тест, баллы & $28,1(21,0 ; 39)$ & $30,0(27,0 ; 36,0)$ & $>0,05$ \\
\hline Одышка по шкале mMRC, баллы & $3,4(3 ; 4)$ & $3,5(3 ; 4)$ & $>0,05$ \\
\hline Одышка по шкале Borg, баллы & $8,7(8,0 ; 10,0)$ & $8,7(8,0 ; 10,0)$ & $>0,05$ \\
\hline $\mathrm{OФB}, \%$ & $31,3(20 ; 59)$ & $32,8(13,0 ; 56,0)$ & $>0,05$ \\
\hline $\mathrm{pO}_{2}$, мм рт. ст. & $58,69(33,5 ; 72,4)$ & $59,6(46,3 ; 76,9)$ & $>0,05$ \\
\hline $\mathrm{pCO}_{2}$, мм рт. ст. & $45,63(28,6 ; 65,8)$ & $45,6(26,1 ; 74,3)$ & $>0,05$ \\
\hline $\mathrm{pH}$ & $7,41(7,36 ; 7,47)$ & $7,40(7,38 ; 7,44)$ & $>0,05$ \\
\hline Поверхностная миография четырехглавой мышцы бедра, мкВ & $204,06(55,1 ; 435)$ & $194,3(58 ; 443)$ & $>0,05$ \\
\hline Шагомер, кол-во шагов & $295(38,0 ; 700,0)$ & $220(45,0 ; 651)$ & $>0,05$ \\
\hline
\end{tabular}

Примечание: * — все данные представлены в виде $\mathrm{Me}\left(\mathrm{Q}_{1} ; \mathrm{Q}_{3}\right) ; p$ — достоверность различий между двумя группами.

Таблица 2. Сравнение эфффективности ЭМС в основной группе и группе сравнения

\begin{tabular}{|l|c|c|c|}
\hline \multicolumn{1}{|c|}{ Параметры } & Основная группа $(n=18)$ & Группа контроля $(n=18)$ & $p$ \\
\hline \multicolumn{3}{|c|}{ Качество жизни и SpО } \\
\hline
\end{tabular}

Примечание: * — все данные представлены в виде $\mathrm{Me}\left(\mathrm{Q}_{1} ; \mathrm{Q}_{3}\right) ; p$ - достоверность различий между двумя группами. 


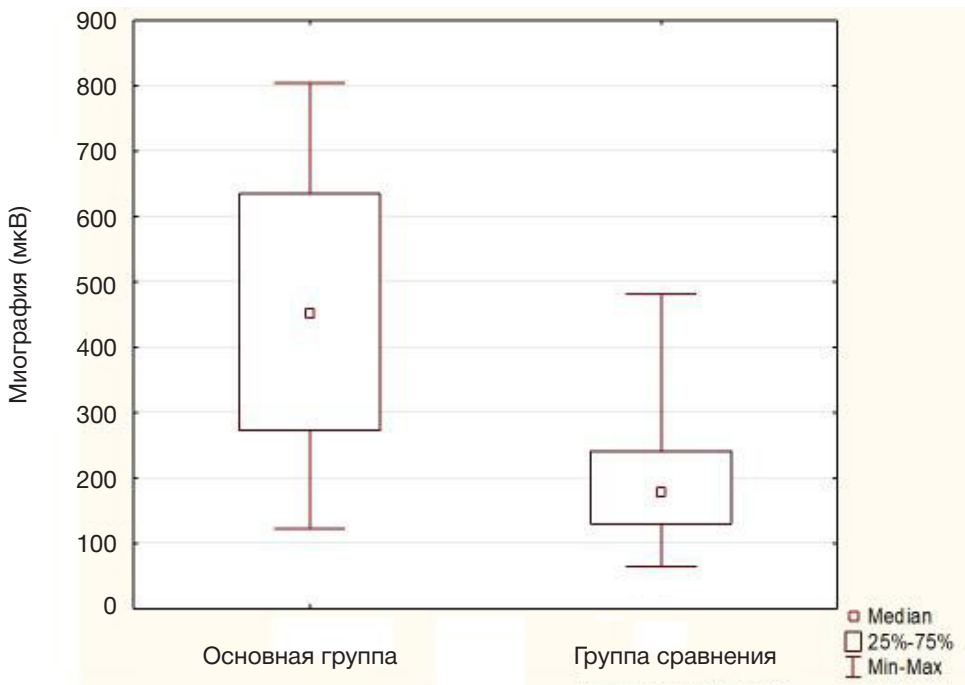

Рис. 2. Показатели миографии на 10 сутки (сравнение двух независимых групп количественных признаков с применением U-теста Манна-Уитни)

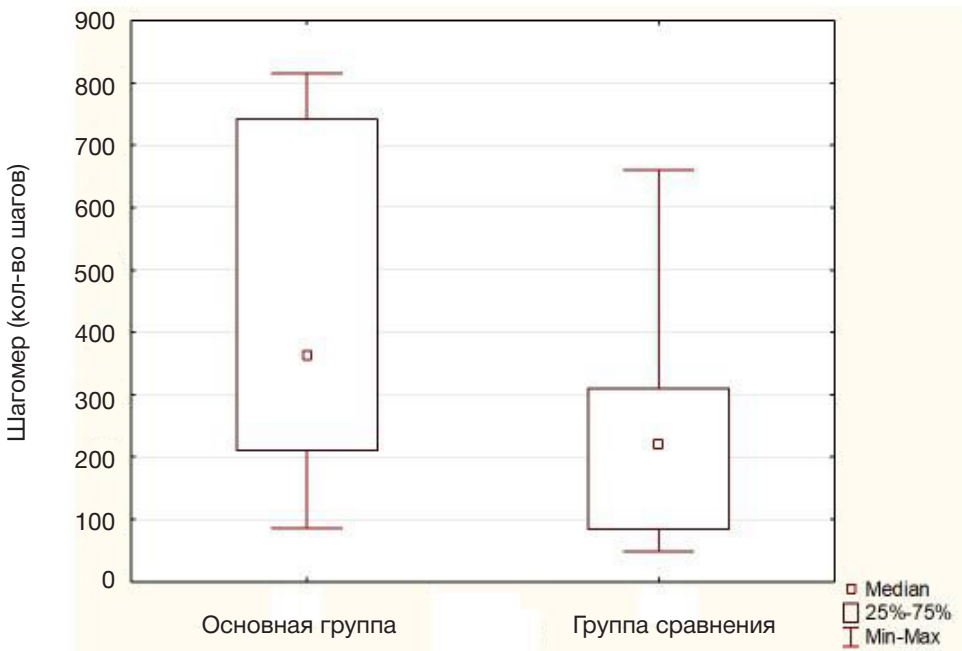

Рис. 3. Показатели шагомера на 10 сутки (сравнение двух независимых групп количественных признаков с применением U-теста Манна-Уитни)

\section{ВЫВОДЫ}

По результатам работы можно сделать вывод, что электромиостимуляция четырехглавой мышцы бедра может стать эффективной альтернативой тренировкам у больных с тяжелым обострением ХОБЛ. Данный метод можно использовать в качестве одного из компонентов программы легочной реабилитации у пациентов с ХОБЛ, не способных выполнять физические упражнения.

\section{Литература}

1. Чучалин А. Г., Авдеев С. Н., Айсанов З. Р., Белевский А. С., Лещенко И. В., Мещерякова Н. Н., Овчаренко С. И., Шмелев Е. И. Федеральные клинические рекомендации по диагностике и лечению хронической обструктивной болезни легких. Пульмонология. 2014; (3): 15-54.

2. Deckamer $\mathrm{V}$, Vogelmeier $\mathrm{C}$. Global strategy for the diagnosis, management, and prevention of chronic obstructive pulmonary disease. Global Initiative for Chronic Obstructive Lung Disease. 2015: 1-44.

3. Авдеев С. Системные эфффекты у больных ХОБЛ. Врач. 2006; 12: 3-8.

4. Перцева Т. А., Санина Н. А. Выраженность системных воспалительных реакций у больных хронической обструктивной болезнью легких. Пульмонология. 2013; (1): 38-41.

5. Barreiro E, Gea J. Molecular and biological pathways of skeletal muscle dysfunction in chronic obstructive pulmonary disease.

Chron Respir Dis. 2016; 13 (3): 297-311.

6. Мухарлямов Ф. Ю., Сычева М. Г., Рассулова М. А., Разумов А. Н. Пульмонологическая реабилитация: современные программы и перспективы. Пульмонология. 2013; 6: 99-105.

7. Ont Health Technol Assess Ser. Pulmonary Rehabilitation for Patients With Chronic Pulmonary Disease (COPD): an EvidenceBased Analysis.. 2012; 12 (6): 1-75

8. Мещерякова Н. Н., Белевский А. С., Черняк А. В., Лебедин Ю. С. Влияние методов легочной реабилитации на маркеры системного воспаления и уровень тестостерона в крови у больных хронической обструктивной болезнью легких. Пульмонология. 2011; 2: 81-86.

9. Rong-chang Chen, Xiao-ying Li. Effectivness of neuromuscular electrical stimulation for the rehabilitation of moderate-to-severe COPD: a meta-analysis. Chron Respir Dis. 2016; 13 (3): 297-311. 10. Abdellaoui A, Préfaut C, Gouzi F, Couillard A, Coisy-Quivy M, 
Hugon G, et al. Skeletal muscle effects of electrostimulation after COPD exacerbation: a pilot study. Europ Resp J. 2011; 38: 781-8.

11. Сандухадзе Б. Р. Возможности кардиосинхронизированной электромиостимуляции в лечении хронической сердечной недостаточности у больных на фоне ИБС [диссертация]. М.: 2009.

12. Barreiro E, Gea J. Molecular and biological pathways of skeletal muscle dysfunction in chronic obstructive pulmonary disease. Chron Respir Dis. 2016; 13 (3): 297-311.

13. Windholz T, Swanson T, Vanderbyl BL, Jagoe RT. The feasibility and acceptability of neuromuscular electrical stimulation to improve exercise performance in patients with advanced cancer: a pilot study. BMC Palliat Care. 2014; 13: 23.

14. Fischer A, Spiegl M, Altmann K. Muscle mass, strength and functional outcomes in critically ill patients after cardiothoracic surgery: does neuromuscular electrical stimulation help? The Catastim 2 randomized controlled trial. Crit Care. 2015; 20: 30.

15. Abdulai RM, Jensen TJ, Patel NR, Polkey MI, Jansson P, Celli BR, Rennard SI. Deterioration of Limb Muscle Function during Acute Exacerbation of Chronic Obstructive Pulmonary Disease. Am J Resp Crit Care Medicine. 2017; 197 (4): 433-49.

\section{References}

1. Chuchalin AG, Avdeev SN, Ajsanov ZR, Belevskij AS, Leshhenko IV, Meshherjakova NN, Ovcharenko SI, Shmelev El. Federal'nye klinicheskie rekomendacii po diagnostike i lecheniju hronicheskoj obstruktivnoj bolezni legkih. Pul'monologija. 2014; (3): 15-54.

2. Deckamer V, Vogelmeier $\mathrm{C}$. Global strategy for the diagnosis, management, and prevention of chronic obstructive pulmonary disease. Global Initiative for Chronic Obstructive Lung Disease. 2015: 1-44.

3. Avdeev S. Sistemnye jeffekty u bol'nyh HOBL. Vrach. 2006; 12: 3-8.

4. Perceva TA, Sanina NA. Vyrazhennost' sistemnyh vospalitel'nyh reakcij u bol'nyh hronicheskoj obstruktivnoj bolezn'ju legkih. Pul'monologija. 2013; (1): 38-41.

5. Barreiro E, Gea J. Molecular and biological pathways of skeletal muscle dysfunction in chronic obstructive pulmonary disease. Chron Respir Dis. 2016; 13 (3): 297-311.

6. Muharljamov FJu, Sycheva MG, Rassulova MA, Razumov AN. Pul'monologicheskaja reabilitacija: sovremennye programmy perspektivy. Pul'monologija. 2013; 6: 99-105.

7. Ont Health Technol Assess Ser. Pulmonary Rehabilitation for Patients With Chronic Pulmonary Disease (COPD): an EvidenceBased Analysis.. 2012; 12 (6): 1-75

8. Meshherjakova NN, Belevskij AS, Chernjak AV, Lebedin JuS. Vlijanie metodov legochnoj reabilitacii na markery sistemnogo vospalenija i uroven' testosterona v krovi u bol'nyh hronichesko

obstruktivnoj bolezn'ju legkih. Pul'monologija. 2011; 2: 81-86.

9. Rong-chang Chen, Xiao-ying Li. Effectivness of neuromuscular electrical stimulation for the rehabilitation of moderate-to-severe COPD: a meta-analysis. Chron Respir Dis. 2016; 13 (3): 297-311. 10. Abdellaoui A, Préfaut C, Gouzi F, Couillard A, Coisy-Quivy M, Hugon G, et al. Skeletal muscle effects of electrostimulation after COPD exacerbation: a pilot study. Europ Resp J. 2011; 38: 781-8.

11. Sanduhadze BR. Vozmozhnosti kardiosinhronizirovannoj jelektromiostimuljacii $\vee$ lechenii hronicheskoj serdechnoj nedostatochnosti u bol'nyh na fone IBS [dissertacija]. M.: 2009.

12. Barreiro E, Gea J. Molecular and biological pathways of skeletal muscle dysfunction in chronic obstructive pulmonary disease. Chron Respir Dis. 2016; 13 (3): 297-311.

13. Windholz T, Swanson T, Vanderbyl BL, Jagoe RT. The feasibility and acceptability of neuromuscular electrical stimulation to improve exercise performance in patients with advanced cancer: a pilot study. BMC Palliat Care. 2014; 13: 23.

14. Fischer A, Spiegl M, Altmann K. Muscle mass, strength and functional outcomes in critically ill patients after cardiothoracic surgery: does neuromuscular electrical stimulation help? The Catastim 2 randomized controlled trial. Crit Care. 2015; 20: 30.

15. Abdulai RM, Jensen TJ, Patel NR, Polkey Ml, Jansson P, Celli BR, Rennard SI. Deterioration of Limb Muscle Function during Acute Exacerbation of Chronic Obstructive Pulmonary Disease. Am J Resp Crit Care Medicine. 2017; 197 (4): 433-49. 